\title{
Role of Anxiety, Depression and Anger in Eating Disorders- Structural Model
}

\author{
Seyyed Mohammad Moosavi ${ }^{1}$, Fatemeh Amini ${ }^{2, ~ * ~}$ \\ ${ }^{1}$ Department of Psychiatry, Mazandaran University of Medical Sciences, Sari, Iran \\ ${ }^{2}$ Department of Psychology, Islamic Azad University of Sari, Sari, Iran
}

Email address:

Fatemeh2000@gmail.com (F. Amini)

${ }^{*}$ Corresponding author

\section{To cite this article:}

Seyyed Mohammad Moosavi, Fatemeh Amini. Role of Anxiety, Depression and Anger in Eating Disorders- Structural Model. American Journal of Psychiatry and Neuroscience. Vol. 5, No. 4, 2017, pp. 40-44. doi: 10.11648/j.ajpn.20170504.11

Received: June 18, 2017; Accepted: July 19, 2017; Published: August 14, 2017

\begin{abstract}
Aim of current study was to offer structural model regarding role of anxiety, depression and anger in eating disorder. Noting morbidity and mushrooming trend of eating disorder in clinical settings and its antagonistic features among afflicted individuals, with respect to clinical implication and, theoretical dimensions, conducting current study seemed vital. Materials and Methods: This study was descriptive-correlational study. Statistical society of current study, consisted of 750 individuals with $\mathrm{BMI} \geq 25$, who were admitted to nutrition clinic at Sari General Hospital (Sari, Iran). Sample size was specified as 226 according to Krejcie and Morgan Table. We used DASS-20 with respect to depression, anxiety and stress assessment; Spielberger's State-Trait Anger Expression Inventory in clinical patients with respect to anger assessment, EDDS (Eating disorder diagnostic scale) regarding assessment of eating disorders (anorexia nervosa, bulimia nervosa and binge eating disorder). With respect to data analyze, SPSS-22 and structural equation modeling and regression model via Lisrel test were used. Results: According to results of current study, anxiety, depression and aggression maintain positive and influential roles with respect to anorexia nervosa, bulimia nervosa and binge eating disorder.
\end{abstract}

Keywords: Anxiety, Depression, Aggression, Anorexia Nervosa, Bulimia Nervosa, Binge Eating Disorder

\section{Introduction}

Eating disorders, which are associated with a host of adverse medical morbidities, negative psychological sequelae, and considerable reductions in quality of life, should be diagnosed and treated promptly. However, primary care physicians may find it uniquely challenging to detect eating disorders in their early stages, before obvious physical problems arise and while psychological symptoms are subtle. Although psychological symptoms may dominate the presentation, the physician is an integral member of the treatment team and is in a unique role to diagnose and treat eating disorders [1].

Health service utilization among adults with eating disorders is high, and hospitalization expenses are on the rise [2]. Among adults with eating disorders, at least half had their disorder first diagnosed by their primary care physicians $[3,4]$. Although primary care physicians are in a unique role to diagnose and treat eating disorders, reviews of medical education frequently show a lack of adequate training in their identification and treatment, and hence health care professionals may only detect an eating disorder once substantial medical and psychological consequences have developed. [4, 5] Eating disorders are an important cause of physical and psychosocial morbidity in adolescent girls and young adult women. They are much less frequent in men. Eating disorders are divided into three diagnostic categories: anorexia nervosa, bulimia nervosa, and the atypical eating disorders. However, the disorders have many features in common and patients frequently move between them, so for the purposes of this Seminar we have adopted a transdiagnostic perspective. The cause of eating disorders is complex and badly understood. There is a genetic predisposition, and certain specific environmental risk factors have been implicated. Research into treatment has focused on bulimia nervosa, and evidence-based management of this disorder is possible. A specific form of cognitive behaviour 
therapy is the most effective treatment, although few patients seem to receive it in practice. Treatment of anorexia nervosa and atypical eating disorders has received remarkably little research attention [6].

(1) Anorexia Nervosa (AN)

The prevalence of $\mathrm{AN}$ is approximately $0.5 \%$ to $1 \%$ and is highest among adolescent girls and young women. Anorexia nervosa is characterized by an abnormally low body weight (at least $15 \%$ below what would be expected), a corresponding fear of weight gain, and an undue emphasis on weight and shape in self-evaluation [7]. Although amenorrhea (ie, loss of 3 consecutive menstrual cycles) is currently required for the diagnosis, the importance of this symptom is unclear, and as such, the eating disorders workgroup of the Diagnostic and Statistical Manual of Mental Disorders (DSM) (Fifth Edition) has strongly considered removing it as a criterion for AN [8] Anorexia nervosa can be classified into 2 subtypes: the restricting subtype and the binge-eating/purging subtype. Patients with AN who rarely binge-eat or purge but maintain a fairly regular pattern of caloric restriction may be classified as having the restricting subtype, whereas those who regularly engage in binge eating and/or compensatory behavior to prevent weight gain will be diagnosed as having the bingeeating/purging subtype [7]. Many of those with the restricting subtype will eventually develop binge eating, with at least one-third of patients crossing over into BN [13]. Crossover to binge eating and BN typically occurs within the first 5 years of the illness [13]. Women with AN who develop BN are likely to relapse back into AN [13].

The outcomes associated with AN are poor, with only a $35 \%$ to $85 \%$ recovery rate and a protracted recovery, ranging from 57 to 79 month [14]. Not only can AN evolve into a chronic condition, it is one of the most medically serious psychiatric disorders $[15,16]$ People with AN are affected by the physical consequences of the severe weight loss, along with psychological comorbid conditions that contribute to mortality, [17] with suicides representing a large portion of the deaths from AN. [18] Depression, a consequence of poor caloric intake and low weight, is frequently comorbid with $\mathrm{AN}$ and often resolves with re-feeding [16] Anxiety symptoms are common and often precede the development of the illness [19].

\section{(2) BULIMIA NERVOSA}

The clinician will encounter patients with $\mathrm{BN}$ more often than those with $\mathrm{AN}$, because $\mathrm{BN}$ has a higher prevalence among women $(1.0 \%-1.5 \%)$. However, often secretive and lacking obvious physical stigmata such as emaciation, patients with $\mathrm{BN}$ may avoid detection, with only a minority seeking treatment (12-17) The modal patient is a woman aged 16 to 22 years; however, the physician may encounter $\mathrm{BN}$ in older patients. Bulimia nervosa can be classified into 2 subtypes: the purging type, which is characterized by episodes of binge-eating (an inordinately large amount of food, in a short period of time, in an out-of-control fashion), followed by compensatory behavior, such as self-induced vomiting, laxative abuse, and diuretic abuse; and the nonpurging type, which is characterized by excessive exercise, fasting, or strict diets. 5 As with $\mathrm{AN}$, patients with BN may place undue emphasis on their body shape and live in fear of gaining weight [10]. Currently, if binge eating and purging occur in the context of low weight and amenorrhea, $\mathrm{AN}$ is diagnosed. Although crossover from $\mathrm{AN}$ to $\mathrm{BN}$ is common, crossover from $\mathrm{BN}$ to $\mathrm{AN}$ is relatively rare unless the patient was originally diagnosed as having AN [17-19].

\section{(3) BINGE-EATING DISORDER}

Binge-eating disorder is characterized by the consumption of large amounts of food in a 2-hour time period, accompanied by a perceived loss of control. 5 Additional symptoms include feeling uncomfortably full, eating rapidly, eating alone, eating when not hungry, and feeling disgusted afterward [17-19] Unlike BN, compensatory behavior (eg, vomiting, laxative abuse) does not accompany these binge episodes. 5 When diagnosing BED, the clinician should take care to differentiate it from overeating. Overeating episodes often occur at social functions, where abundant food is readily available, the mood is relaxed or positive, and other people are also overeating, whereas binge episodes typically are secretive and occur in the context of negative mood and all-ornothing thinking. Primary care physicians may elect to screen for BED using the Eating Attitudes Test, the most widely used eating disorder screening tool [8-10]. Alternatively, the Eating Disorder Diagnostic Scale is a 22item, self-report inventory created to diagnose $\mathrm{AN}, \mathrm{BN}$, and BED in accordance with $D S M-I V$ criteria. 8 However, the Questionnaire on Eating and Weight Patterns-Revised, which generates diagnostic information regarding BED, in addition to information regarding dieting and weight history, has been widely used with diverse community and clinical samples, including severely obese candidates for bariatric surgery [10-13-19].

All in all, in current study we aimed to define the role of depression, anxiety and aggression in Eating disorders via structural model approach. We would discuss the main eating disorders seen in primary care, including anorexia nervosa (AN), bulimia nervosa (BN), binge-eating disorder (BED).

\section{Materials and Methods}

This study was descriptive-correlational study. Statistical society of current study, consisted of 750 individuals with $\mathrm{BMI} \geq 25$, who were admitted to nutrition clinical at Sari General Hospital (Sari, Iran). Sample size was specified as 226 according to Krejcie and Morgan Table. We used DASS20 with respect to depression, anxiety and stress assessment; Spielberger's State-Trait Anger Expression Inventory in clinical patients with respect to anger assessment, EDDS (Eating disorder diagnostic scale) regarding assessment of eating disorders (anorexia nervosa, bulimia nervosa and binge eating disorder). With respect to data analyze, SPSS-22 and structural equation modeling and regression model via Lisrel test were used.

(1) DASS 
DASS, the Depression Anxiety Stress Scales, [20] is made up of 42 self-report items to be completed over five to ten minutes, each reflecting a negative emotional symptom [21], Each of these is rated on a four-point Likert scale of frequency or severity of the participants' experiences over the last week with the intention of emphasizing states over traits. These scores ranged from 0 , meaning that the client believed the item "did not apply to them at all", to 3 meaning that the client considered the item to "apply to them very much, or most of the time". It is also stressed in the instructions that there are no right or wrong answers. The sum of the relevant 14 items for each scale constitute the participants' scores for each of Depression, Anxiety and Stress, [21] including items such as "I couldn't seem to experience any positive feeling at all", "I was aware of the dryness of my mouth" and "I found it hard to wind down" in the respective order of the scales. The order of the 42 items has been randomized so that items of the same scale are not clustered together. Each of the scales is then broken down into subscales comprising two to five items each.

The Depression scale has subscales assessing dysphoria, hopelessness, devaluation of life, self-deprecation, lack of interest/involvement, anhedonia and inertia.

The Anxiety scale assesses autonomic arousal, skeletal muscle effects, situational anxiety and subjective experience of anxious affect.

The Stress scale's subscales highlight levels of non-chronic arousal through difficulty relaxing, nervous arousal and being easily upset/agitated, irritable/over-reactive and impatient [20].

(2) State-Trait Anger Expression Inventory-

The State-Trait Anger Expression Inventory-2 (STAXI-2) assesses various areas of anger and the traits of experiencing anger. A common use for the STAXI is to collect information for people with anger issues and for creating treatment plans. Participants are asked to respond to 57 items using a 4-point scale ("Not at all" to "Almost Always"). Approximately 12 to 15 minutes is required for completion. STAXI is the revised edition which had only included 44 -items. The StateTrait Anger Expression Inventory is a well-known instrument and it has supported data for relatively high reliability and validity. In the authors test manuals, the psychometric properties included high alpha coefficients for internal reliability for all subscales except for the Trait Anger Scale/Angry Reaction (0.73-0.76). There is extensive showing of validity for the original versions of STAXI but none for the current edition. Concurrent validity of the original STAXI is strongly presented with correlations with the Multiphasic Inventory (Hostility and Over Hostility Scales), Buss-Durkee Hostility Inventory, and the Eysenck Questionnaire (Psychoticism and Neuroticism).

(3) The Eating Disorder Diagnostic Scale

(EDDS) is a 22 item self-report questionnaire that assesses the presence of three eating disorders; anorexia nervosa, bulimia nervosa and binge eating disorder. It was adapted by Stice et al. in 2000 from the validated structured psychiatric interview: The Eating Disorder Examination (EDE) and the eating disorder module of the Structured Clinical Interview for DSM-IV (SCID)16 [22]. In follow up studies of the reliability and validity of the EDDS it was shown to be sufficiently sensitive to detect the effects of eating disorders prevention programs, response to such programs and the future onset of eating disorder pathology and depression. The EDDS shows both full and sub threshold diagnoses for anorexia nervosa, bulimia nervosa and binge eating disorder. EDDS is a continuous eating disorder symptom composite score [23]. The PhenX Toolkit uses the EDDS for as an Eating Disorders Screener protocol [24].

\section{Results}

According to results of current study, as predicted anxiety, depression, anger, maintain decisive role regarding pathogenesis of Anorexia Nervosa.

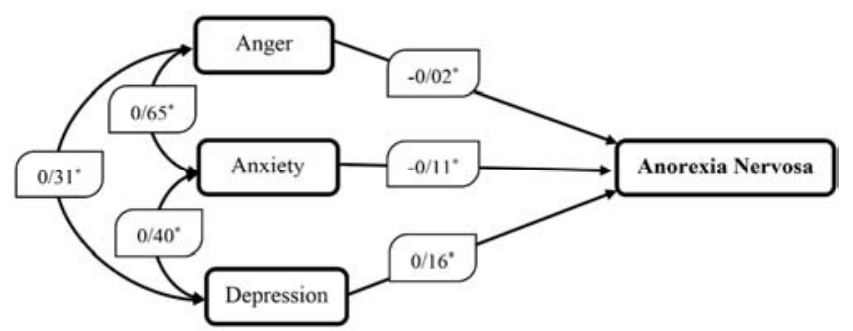

Figure 1. Analyzing Dynamic of Anger, Anxiety and Depression with Respect to Anorexia Nervosa.

Figure 1, delineates dynamic and valence of Anger, Anxiety and Depression with respect to pathogenesis of AN.

With respect to results of current study, as predicted anxiety, depression, anger, maintain decisive role regarding pathogenesis of Bulimia Nervosa.

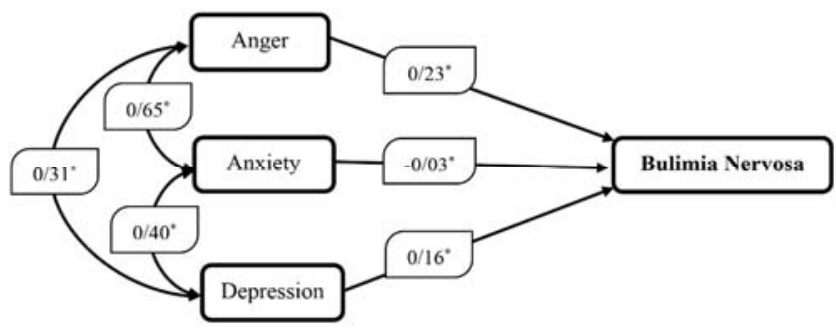

Figure 2. Analyzing Dynamic of Anxiety, Depression and Anger with Respect to Bulimia Nervosa.

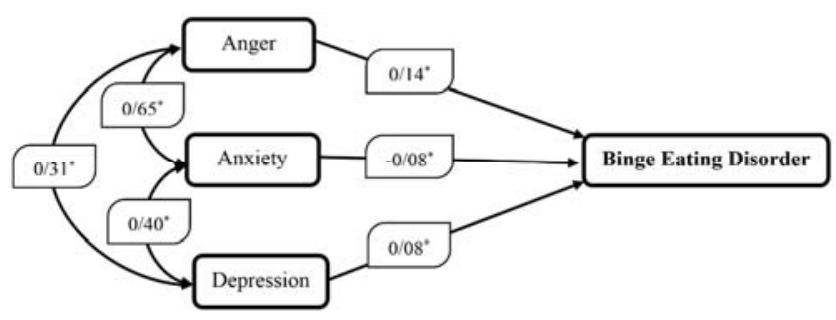

Figure 3. Analyzing Dynamic of Anxiety, Depression and Anger with respect to Binge Eating Disorder.

Figure 2, delineates dynamic and valence of Anger, Anxiety and Depression with respect to pathogenesis of AN. 
According to results of current study, as predicted anxiety, depression, anger, maintain decisive role regarding pathogenesis of BN.

All in all, Figure 2 delineates dynamic and valence of Anger, Anxiety and Depression with respect to pathogenesis of BEA.

\section{Discussion}

Noble results of current study is that it propose a quantitative, schematic model with respect to dynamic of Anger, Anxiety and Depression regarding pathogenesis of anorexia nervosa, bulimia nervosa and binge eating disorder.

In a same manner, Matilda and Colleagues (2013), looked at dynamic of eating disorders and other comorbid psychiatric conditions. In their study, they looked at dynamic between Alexithymia and eating disorders. In summary, there is a robust body of literature showing that individuals with eating disorders experience difficulties identifying and describing emotions. Given these affectrelated difficulties, it is important that eating disorder treatment protocols also incorporate a focus on emotions and emotion regulation [22]. Consistent with the result of current study, Casper (1998) studied relationship of depression and eating disorder. Aforementioned study, confirm the prominence of depressive symptoms and depressive disorders in eating disorders. Other psychiatric syndromes, which occur with less frequency, such as anxiety disorders and obsessive-compulsive disorders in anorexia nervosa, or personality disorders, anxiety disorders, and substance abuse in bulimia nervosa, also play an important role in the development and maintenance of eating disorders. Since few studies have controlled for starvation-induced physical, endocrine, or psychological changes, which mimic the symptoms considered diagnostic for depression, further research will be needed. The evidence for a shared etiology is not compelling for anorexia nervosa and is at most suggestive for bulimia nervosa. Since in contemporary cases dieting-induced weight loss is the principal trigger, women with self-critical or depressive features will be disproportionately recruited into eating disorders. The model that fits the data best would accommodate a relationship between eating disorders and the full spectrum of depressive disorders from no depression to severe depression, with somewhat higher rates of depression in bulimic anorectic and bulimia nervosa patients than in restricting anorexia nervosa patients, but the model would admit a specific pathophysiology and psychopathology in each eating disorder [23]. Additionally, Hughes and Collegues (2013) studied eating disorders with and without comorbid depression and anxiety. They concluded that, Depression and anxiety were differentially related to clinical characteristics of EDs. The findings have implications for understanding the relations between these disorders and their potential to impact outcome of ED treatments [24].

\section{References}

[1] Leslie A. Sim, PhD, Donald E. Mc Alpine, MD, Karen B. Grothe, PhD, Susan M. Himes, PhD, Richard G. Cockerill, BA, and Matthew M. Clark, PhD. (2010). Identification and Treatment of Eating Disorders in the Primary Care Setting. Available:

https:/www.ncbi.nlm.nih.gov/pmc/articles/PMC2912736/. Last accessed Feb 2017.

[2] Mond JM, Hay PJ, Rodgers B, Owen C. Health service utilization for eating disorders: findings from a communitybased study. Int J Eat Disord. 2007; 40 (4):399-408 [PubMed].

[3] Hudson JI, Hiripi E, Pope HG, Jr, Kessler RC. The prevalence and correlates of eating disorders in the national comorbidity survey replication. Biol Psychiatry 2007; 61 (3):348-358 [PMC free article] [PubMed].

[4] Walsh JM, Wheat ME, Freund K. Detection, evaluation and treatment of eating disorders: the role of the primary care physician. J Gen Intern Med. 2000; 15 (8): 577-590 [PMC free article] [PubMed].

[5] Currin L, Schmidt U, Waller G. Variables that influence diagnosis and treatment of the eating disorders within primary care settings: a vignette study. Int J Eat Disord. 2007; 40 (4): 257-262 [PubMed].

[6] Lancet. 2003 Feb 1; 361(9355):407-16. (2003). Eating disorders. Available:

https://www.ncbi.nlm.nih.gov/pubmed/12573387. Last accessed Jan 2017.

[7] American Psychiatric Association Diagnostic and Statistical Manual of Mental Disorders, Fourth Edition, Text Revision $(D S M-I V-T R) \quad$ Washington, DC: American Psychiatric Association; 2000.

[8] Attia E, Roberto C. Should amenorrhea be a criterion for AN. Int J Eat Disord. 2009; 42 (7):581-589 [PubMed].

[9] Perry L, Morgan J, Reid F, et al. Screening. for symptoms of eating disorders: reliability of the SCOFF screening tool with written compared to oral delivery. Int J Eat Disord. 2002; 32 (4):466-472 [PubMed].

[10] Stice E, Telch C, Rizvi S. Development and validation of the Eating Disorder Diagnostic Scale: a brief self-report measure of anorexia, bulimia, and binge eating disorder. Psychol Assess 2000; 12 (2): 123-131 [PubMed].

[11] Garner DM, Olmstead MP, Polivy J. Development and validation of a multidimensional eating disorder inventory for anorexia nervosa and bulimia. Int J Eat Disord. 1983; 2 (2): 15-34.

[12] Spitzer RL, Yanovski SZ, Marcus MD. Questionnaire on Eating and Weight Patterns-Revised (QEWP-R) New York, NY: New York State Psychiatric Institute; 1993.

[13] Eddy KT, Dorer DJ, Franko DL, Tahilani K, ThompsonBrenner H, Herzog DB. Diagnostic crossover in anorexia nervosa and bulimia nervosa: implications for DSM-V. Am J $\begin{array}{lll}\text { Psychiatry 2008; } 165 & \text { (2):245-250 [PMC free article] }\end{array}$ [PubMed]. 
[14] Strober M, Freeman R, Morrell W. The long-term course of severe anorexia nervosa in adolescents: survival analysis of recovery, relapse, and outcome predictors over 10-15 years in a prospective study. Int J Eat Disord. 1997; 22 (3):339-360 [PubMed].

[15] Katzman DK. Medical complications in adolescents with anorexia nervosa: a review of the literature. Int J Eat Disord. 2005; 37 (suppl): S52-S59 [PubMed].

[16] Sullivan PF. Mortality in anorexia nervosa. Am J Psychiatry 1995; 152 (7):1073-1074 [PubMed].

[17] Herzog DB, Keller MB, Sacks NR, Yeh CJ, Lavori PW. Psychiatric comorbidity in treatment-seeking anorexics and bulimics. J Am Acad Child Adolesc Psychiatry 1992; 31 (5):810-818 [PubMed].

[18] Fairburn CG, Harrison PJ. Eating disorders. Lancet 2003; 361 (9355):407-416 [PubMed].

[19] Godart N, Berthoz S, Rein Z, et al. Does the frequency of anxiety and depressive disorders differ between diagnostic subtypes of anorexia nervosa and bulimia? Int $J$ Eat Disord. 2006; 39 (8):772-778 [PubMed].

[20] Lovibond, S. H; Lovibond, P. F. (1995). "Manual for the Depression Anxiety Stress Scales (2nd ed). Sydney:
Psychology Foundation. (Available from The Psychology Foundation, Room 1005 Mathews Building, University of New South Wales, NSW 2052, Australia)

[21] Charles Spielberger. (1999). State-Trait Anger Expression Inventory-2 (STAXI-2). Available:

http://www.statisticssolutions.com/psychological-personalitystate-trait-anger-expression-inventory-2-staxi-2/. Last accessed Jan 2017.

[22] Matilda E Nowakowski, corresponding author 1 Traci Mc Farlane, 1, 2 and Stephanie Cassin 1. (2013). Alexithymia and eating disorders: a critical review of the literature. Available: https:/www.ncbi.nlm.nih.gov/pmc/articles/PMC4081716/. Last accessed May-2017.

[23] Casper RC. (1998). Depression and eating disorders. Available: https://www.ncbi.nlm.nih.gov/pubmed/9809221. Last accessed May-2017.

[24] Hughes EK, Goldschmidt AB, Labuschagne Z, Loeb KL, Sawyer SM, Le Grange D. (2013). Eating disorders with and without comorbid depression and anxiety: similarities and differences in a clinical sample of children and adolescents. Available: https://www.ncbi.nlm.nih.gov/pubmed/23681932. Last accessed May-2017. 